\title{
IMPACT OF HEADLIGHT GLARE ON PEDESTRIAN DETECTION WITH UNILATERAL CATARACT
}

\author{
Sailaja Manda, Rachel Castle, Alex D. Hwang, Eli Peli \\ Schepens Eye Research Institute, Massachusetts Eye and Ear, \\ Harvard Medical School, Boston, MA, USA \\ Email: eli_peli@meei.harvard.edu
}

\begin{abstract}
Summary: Detecting pedestrians while driving at night is difficult, and is further impeded by oncoming headlight glare (HLG). Cataracts increase intraocular light scattering, making the task even more challenging. We used a within-subjects repeated measures design to determine the impact of HLG on driving with unilateral cataract. Pedestrian detection performance of six young normal vision (NV) subjects was measured with clear lens glasses and with simulated unilateral cataract (0.8 Bangerter foil) glasses. The subjects drove night-time scenarios in a driving simulator with and without custom simulated headlight glare. With simulated unilateral cataracts, pedestrian detection rates decreased and response times increased with oncoming HLG. We verified these effects with six patients who already underwent cataract surgery for one eye and were scheduled to get cataract surgery in the other eye. We measured their performance before and after the second cataract surgery. The results were similar to those obtained with the simulated unilateral cataract, confirming that a negative impact of HLG persists with untreated cataract in one eye.
\end{abstract}

\section{INTRODUCTION}

Cataract is the clouding of the normally clear crystalline lens in the eye, mostly attributed to aging. Cataracts increase the light scatter within the eye and thereby reduce the contrast of the retinal image (de Waard et al., 1992), putting older drivers at a higher collision risk (Owsley et al., 1999).

Wood et al. (2012) compared nighttime pedestrian detection performance with normal vision to the performance with bilateral cataracts simulating frosted lenses. Their cataract simulation decreased visual acuity by two lines ( 0.2 LogMAR) and contrast sensitivity by about $0.78 \log$ units. Pedestrians walked in place on the far right shoulder (across the oncoming traffic lane in Australia), and stationary halogen lamps were positioned along the course $2.5 \mathrm{~m}$ to the left of the pedestrian in the oncoming lane to mimic the headlamps of a sedan between the driver and the pedestrian. With the headlamps turned on, participants detected significantly more pedestrians in the normal vision condition (57\%) than in the simulated cataract condition (30\%) and from 5.5 fold father distances. Drivers detected nearly twice as many pedestrians when the headlamps were turned off.

In a previous study, we used a custom headlight glare (HLG) simulator integrated with a driving simulator to measure pedestrian detection performance with and without dynamic HLG in nighttime driving scenarios (Hwang et al., 2018). Participants with mild cataract simulation (0.8 Bangerter foils were used bilaterally to reduce visual acuity to $\sim 20 / 35$ and contrast sensitivity to $\sim 1.4 \log C S$ ) missed $6 \%$ of the pedestrians that walked along either side or crossed the road in either direction in the presence of oncoming HLG, compared to $0.5 \%$ without the simulated 
cataract. With simulated cataracts, response times were significantly and meaningfully longer with HLG $(3.4 \pm 1.07 \mathrm{~s})$ than without $(1.23 \pm 0.26 \mathrm{~s})$. In the same study, patients with mild bilateral cataract showed longer reaction times with HLG $(3.77 \pm 1.10 \mathrm{~s})$ than without $(1.81 \pm$ $0.89 \mathrm{~s})$. These prior studies established the negative impact of HLG with bilateral cataract compared to no cataract but did not address unilateral cataract.

Owsley et al. (2001) reported that severely reduced contrast sensitivity in the worse eye (score of 1.25 LogCS or less due to cataract) contributes to a 2.7-fold increase in crash involvement risk. Agramunt et al. (2018) studied self-regulation of driving behavior before and after first eye cataract surgery and after the second eye cataract surgery using an in-car vehicle monitoring device and the Driving Habits Questionnaire (Owsley et al., 1999). Following the first cataract surgery, $30 \%$ of respondents continued to limit driving, while only $10 \%$ continued to selfregulate after the second cataract surgery. These two studies analyzed both daytime and nighttime driving information together and thus the impact of HLG was not studied.

In this study, we first established the effect of HLG on young subjects with and without simulated unilateral cataract to determine the effect of unilateral cataract in individuals whose visual performance is not affected by other age-related factors. We then measured this effect on older patients with unilateral cataract before and after the second cataract surgery. We hypothesized that the light scatter due to unilateral cataract could still worsen pedestrian detection performance in the presence of oncoming HLG.

\section{METHODS}

All participants provided voluntary written informed consent in accordance with the Declaration of Helsinki. The study was approved by the institutional review board at the Massachusetts Eye and Ear.

\section{Participants}

Six normally sighted (NV) subjects ( 2 women) and six unilateral cataract patients (3 women) who were scheduled to undergo cataract surgery completed the study. The mean ages for NV subjects and unilateral cataract patients were $30 \pm 6$ and $64 \pm 13$ years, respectively.

\section{Procedures}

Monocular and binocular visual acuity was measured from $20 \mathrm{ft}$ with the Test Chart 2000 Pro (Thomson Software Solutions, Hatfield, United Kingdom). Contrast sensitivity was measured using the Pelli-Robson contrast sensitivity chart from $1 \mathrm{~m}$. A brief binocular Goldmann perimetry screening test (V4e) verified that the visual field requirement for driving in Massachusetts was met (120 binocularly).

NV subjects were fit with a 0.8 Bangerter foil (Ryser Ophthalmology, Gallen, Germany) affixed to one lens of a plano lens clip-on to simulate unilateral cataract. The foil was placed over the participant's better eye based on visual acuity.

A driving simulator (LE 1500; FAAC Corp., Ann Arbor, MI) equipped with a custom dynamic HLG simulator (Hwang \& Peli, 2013) was used to measure pedestrian detection in realistic night 
driving scenarios. The scenarios were designed to simulate driving at night in a dark urban environment (no street lights). The main light source was the headlights from the participant's car. Pedestrians appeared on the left or right side of the road and either crossed in front of the participant's car or walked along the sidewalk in the same direction as the participant was driving. Participants were instructed to drive at 30 miles per hour following voice instructions (e.g., "turn left at next intersection"), and press the horn on the steering wheel as soon as a pedestrian was detected. Each scenario included 30 encounters with an oncoming car in its own lane, of which 24 included a pedestrian with the oncoming car, and 6 presented only the oncoming car. The scenarios use a custom HLG simulator that presents light (using light emitting diodes and a beam splitter) that is similar to those from the headlamps of an oncoming car at night. These scenarios were used in a pilot study on the impact of HLG with real and simulated bilateral cataracts and are described in detail in Hwang et al. (2018).

Participants first completed an introductory drive to become familiar with the simulator environment, vehicle controls, and study tasks. Once participants demonstrated confidence in their ability to control the vehicle, felt comfortable in the simulator, and showed a clear understanding of the tasks, they started the main study drives.

Participants drove 3 scenarios with and 3 without the headlight glare simulation (HLGY and HLGN, respectively) for each evaluation. The NV subjects completed two evaluations, once with the simulated cataract clip-on and once without (a total of 12 drives) over two test days. The order of HLG presentation (HLGY/HLGN) and viewing condition (with/without the simulated cataract clip-on) were counterbalanced. For patients with unilateral cataract, driving simulator assessments were done before and after cataract surgery for their cataractous eye. Vision measures were repeated following the surgery to ensure that normal vision was achieved. The order of HLG conditions was counterbalanced. The same three scenarios were used in all combinations of HLG and vision conditions.

\section{Analysis}

Average pedestrian detection times were computed for two vision conditions (with or without (simulated) unilateral cataract), two HLG conditions (HLGY or HLGN), and four pedestrian types (walking along the left/right sidewalk or crossing from left/right), and then analyzed in a three-way repeated measures ANOVA. We also calculated the proportion of 'untimely responses' during the pedestrian encounters by applying 1.7 seconds as the theoretical safe response time threshold to avoid a collision, based on the driving speed of 30mph (see Hwang et al., 2018 for details).

\section{RESULTS}

The monocular and binocular visual acuity and contrast sensitivity measures of the study participants with and without simulated or real unilateral cataract are reported in Table 1. The binocular visual acuity and contrast sensitivity measures did not differ significantly between the NV subjects and real patients for the unilateral cataract condition (real vs. simulated). Binocular visual acuity measures were not significantly different for the without (real or simulated) cataract condition but approached significance $[t(10)=2.2, p=0.052]$ for binocular contrast sensitivity measures with cataracts. 
PROCEEDINGS of the Tenth International Driving Symposium on Human Factors in Driver Assessment, Training and Vehicle Design

Table 1. Visual Acuity and Contrast Sensitivity Measures of Normal Vision and Cataract Patients

\begin{tabular}{|c|c|c|c|c|c|c|}
\hline & \multicolumn{3}{|c|}{\begin{tabular}{|l} 
Normal Vision Subjects \\
\end{tabular}} & \multicolumn{3}{|c|}{ Cataract Patients } \\
\hline & \multicolumn{2}{|c|}{$\begin{array}{l}\text { With Unilateral Cataract } \\
\text { Simulation } \\
\end{array}$} & \multirow{2}{*}{$\begin{array}{c}\text { Clear } \\
\text { Lenses } \\
\text { Binocular }\end{array}$} & \multicolumn{2}{|c|}{$\begin{array}{c}\text { Before Surgery } \\
\text { for the Second Eye }\end{array}$} & \multirow{2}{*}{$\begin{array}{c}\text { After } \\
\text { Surgery } \\
\text { Binocular }\end{array}$} \\
\hline & $\begin{array}{c}\text { Eye with } \\
\text { Simulated } \\
\text { Cataract }\end{array}$ & Binocular & & $\begin{array}{c}\text { Eye with } \\
\text { Unilateral } \\
\text { Cataract }\end{array}$ & Binocular & \\
\hline $\begin{array}{l}\text { Visual Acuity } \\
\text { (LogMAR) }\end{array}$ & $0.21(0.09)$ & $-0.06(0.03)$ & $-0.16(0.04)$ & $0.27(0.19)$ & $0.05(0.1)$ & $-0.02(0.05)$ \\
\hline $\begin{array}{c}\text { Contrast Sensitivity } \\
\text { (LogCS) }\end{array}$ & $1.45(0.12)$ & $1.72(0.16)$ & $1.87(0.07)$ & $1.35(0.33)$ & $1.63(0.18)$ & $1.68(0.20)$ \\
\hline
\end{tabular}

NV subjects missed no pedestrians under HLGN and an average of $1 \%( \pm 1 \%)$ under HLGY without the simulated cataract. Figure 1 shows the percentage of pedestrians missed by normal vision subjects and the cataract patients under both vision and HLG conditions.

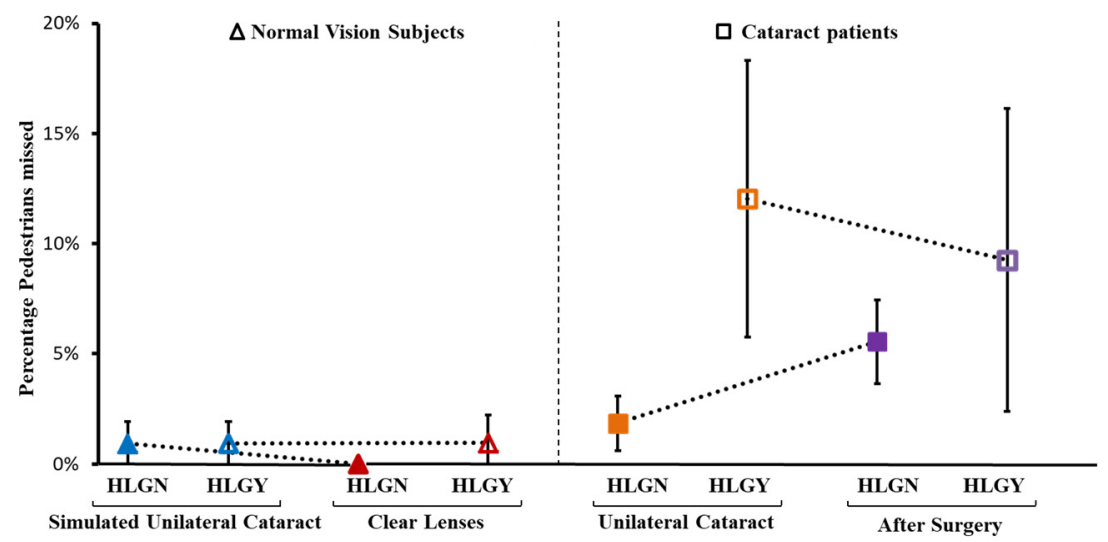

Figure 1. Percentage of pedestrians missed under HLGN and HLGY conditions for NV subjects and Cataract patients with and without simulated or real unilateral cataract. Error bars represent standard errors. Dotted line indicates the effect of vision condition change (i.e. cataract surgery)

The significant effects found in the three-way repeated measures ANOVA $(2 \times 2 \times 4)$ for the pedestrian detection times are given in Table 2. No significant main effect of (or interaction with) the pedestrian type was found.

Table 2. Significant Results Found in the Repeated Measures ANOVA for Response Time

\begin{tabular}{cc|ccccc}
\hline & \multicolumn{2}{c}{ Normal Vision Subjects } & & \multicolumn{2}{c}{ Cataract Patients } \\
\cline { 2 - 3 } \cline { 5 - 6 } \cline { 5 - 6 } & $F$ & $p$ & $\mathbf{0 . 0 5}$ & & 17.3 & 0.01 \\
Main effect of HLG Condition & 6.48 & $<.0 .01$ & & 24.1 & $<0.01$ \\
Main effect of Vision Condition & 23.5 & 7.9 & $\mathbf{0 . 0 4}$ & & 9.7 & $\mathbf{0 . 0 3}$ \\
Interaction & & &
\end{tabular}

Detection times under HLGY were significantly longer than under HLGN with unilateral cataract (real or simulated) and remained significantly longer following the second cataract surgery in patients with unilateral cataract. The impact of HLG was greater before surgery than after for cataract patients while there was no significant difference in response times due to HLG with clear lenses for NV subjects as shown in Fig. 2. 


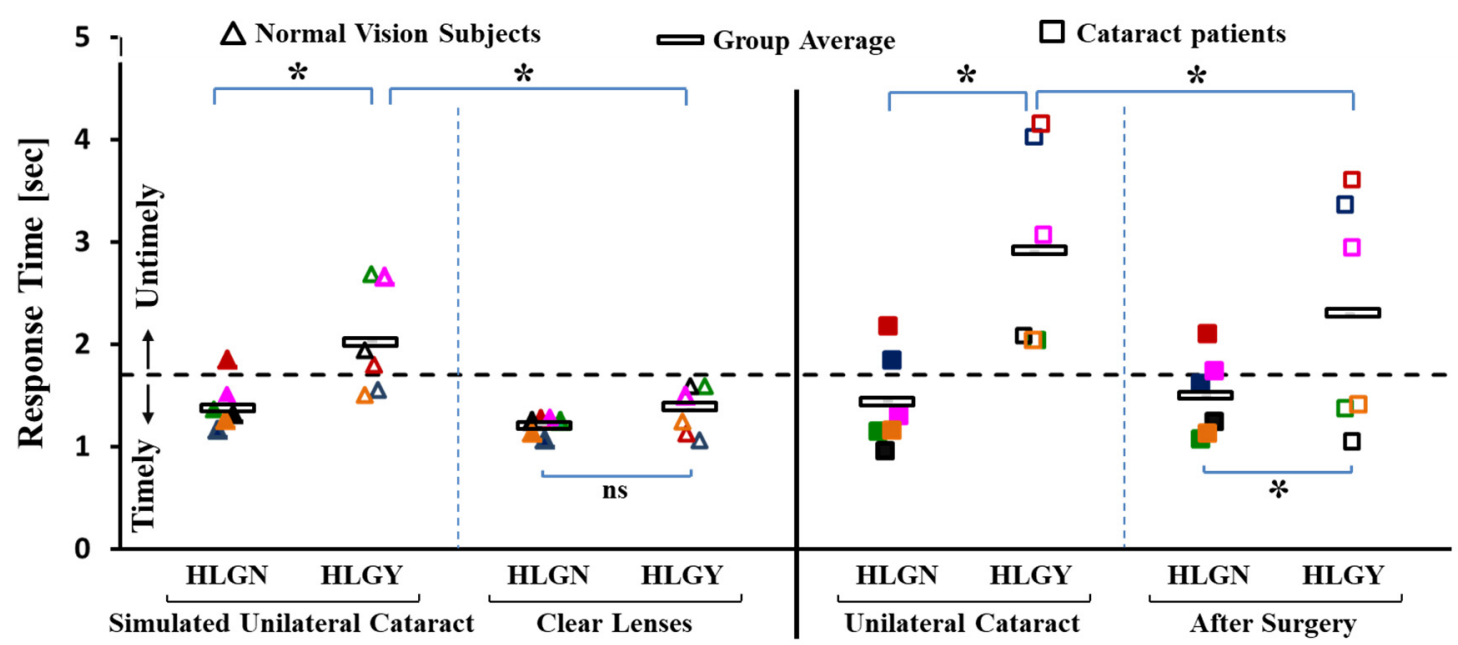

Figure 2. Average of response times for each participant for all encounters under HLGN and HLGY conditions for NV subjects (left) and cataract patients (right) when driving with unilateral (real or simulated) cataract and without cataracts (clear lenses or no cataract). Symbol colors indicate individual subjects in each group.

The average response times to different pedestrian types with and without simulated unilateral cataract are shown in Fig. 3a, and before and after cataract surgery in Fig. 3b. We noted here and have previously observed (Hwang et al., 2018) a pattern of reaction times with respect to pedestrian type.

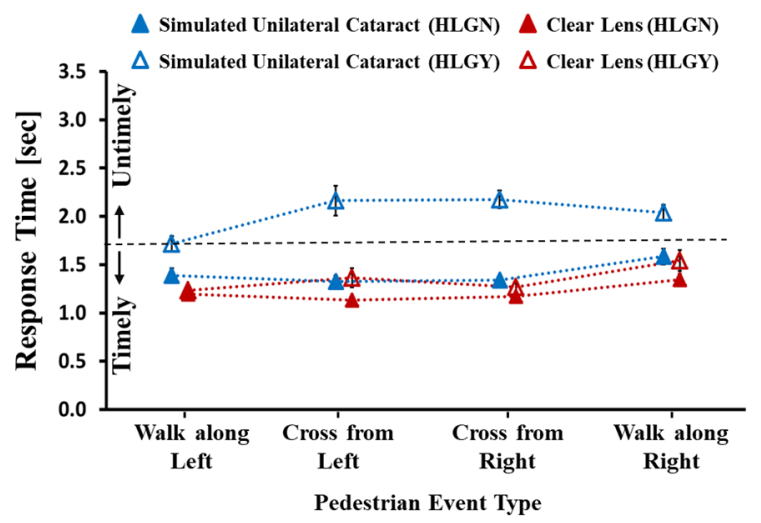

(a)

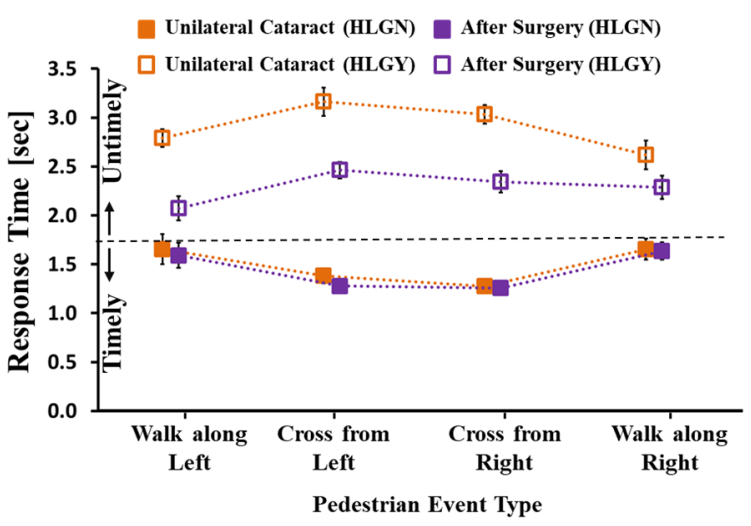

(b)

Figure 3. Average response times for different pedestrian types with and without HLG (HLGY and HLGN, respectively) for (a) NV subjects and (b) Cataract patients. Error bars represent standard deviation.

Under HLGY, all participant groups took longer to respond to pedestrians who walked across the road than pedestrians who walked along the road. Therefore, and in consideration of the relatively small samples in both the groups, we collapsed the data of the four pedestrian events into two action types: crossing vs. walking along the road. The $2 \times 2 \times 2$ ANOVA revealed the same main effects of HLG and cataract as reported above. In addition, a significant interaction between HLG and pedestrian action types was found $[F(1,5)=7.1, p=0.045]$ and $[F(1,5)=$ $7.3, p=0.043]$, for real and simulated cataract, respectively. The post-hoc analysis shows that the response time difference under HLGY was significantly increased only for patients with unilateral cataract $[t(5)=2.99, p=0.03$ ]. For HLGN, both groups took significantly longer [all 
$t \mathrm{~s}(5)>2.63, p<0.05]$ to respond to the pedestrians that walked along the road than to those who crossed the road.

Table 3 shows the means (and standard deviations) of the percentage of untimely responses across all encounters for all HLG and vision conditions. There was a significantly higher rate of untimely responses under HLGY than HLGN for simulated unilateral, but not for clear lenses. Unilateral cataract patients had significantly more untimely responses under HLGY than under HLGN both before and after the cataract surgery.

Table 3. Percentage of Untimely Responses under HLGY and HLGN Conditions

\begin{tabular}{|c|c|c|c|c|c|c|}
\hline & \multicolumn{3}{|c|}{ Normal Vision Subjects } & \multicolumn{3}{|c|}{ Real Cataract Patients } \\
\hline & $\begin{array}{l}\text { Simulated } \\
\text { Unilateral } \\
\text { Cataract }\end{array}$ & $\begin{array}{l}\text { Clear } \\
\text { Lenses }\end{array}$ & p-value & $\begin{array}{l}\text { Real } \\
\text { Unilateral } \\
\text { Cataract }\end{array}$ & $\begin{array}{c}\text { After } \\
\text { Surgery }\end{array}$ & $p$-value \\
\hline HLGY & $49 \%(24 \%)$ & $18 \%(13 \%)$ & $<0.01$ & $72 \%(26 \%)$ & $53 \%(41 \%)$ & 0.04 \\
\hline $\begin{array}{l}\text { HLGN } \\
\text { p-value }\end{array}$ & $\begin{array}{c}17 \%(11 \%) \\
\mathbf{0 . 0 2 4}\end{array}$ & $\begin{array}{c}10 \%(4 \%) \\
0.08\end{array}$ & ns & $\begin{array}{c}25 \%(22 \%) \\
\quad<\mathbf{0 . 0 1}\end{array}$ & $\begin{array}{c}21 \%(24 \%) \\
\mathbf{0 . 0 3}\end{array}$ & ns \\
\hline
\end{tabular}

Data presented as Group Average (Standard Deviation). Bold $\mathbf{p}$ values represent significant differences.

\section{DISCUSSION}

Our results demonstrate a meaningful impact of unilateral cataract on detection of pedestrians in the presence of HLG. There is also an increased risk of collision, as the increase in response time results in significantly more untimely responses with both simulated and real unilateral cataract.

For both groups, the reaction times for events when pedestrians crossed were longer compared to pedestrians walking along the side under HLGY. This is likely due to the proximity of the crossing pedestrian to the glare source. The intra-ocular light scatters from the glare source may obscure these pedestrians until the driver is closer, even with a clear, relatively scatter-free retinal image in the other eye.

In the absence of the glare, all pedestrian were detected much earlier. Older patients with bilateral mild cataract in our previous study (Hwang et al., 2018) demonstrated longer response times under HLGY compared to HLGN, and there was a similar interaction between HLG and pedestrian type. The longer reaction times under both HLGY and HLGN with bilateral cataract compared to our study suggests that cataract surgery for the first eye did improve reaction times. Yet, it is evident that the unilateral cataract continues to affect detection rates and reaction times. Note that visual acuity and contrast sensitivity for the better eye were within normal limits for the patients with unilateral cataract and well within the legal requirements for driving.

All participants in this study had better response times without unilateral impairment, but half of the real patients still showed untimely responses under HLGY following cataract surgery, even though their response times decreased compared to before cataract surgery. This could be attributed to age-related physiological differences affecting night driving performance such as increased intraocular light scatter or delayed dark adaptation. The difficulty with driving at night in the presence of glare is not measurable in the clinic. Also, clinical measures do not relate directly to the risk of crash faced by the drivers (Gruber et al., 2013).

In the US, most cataract surgeries are covered by insurance but in order to qualify for coverage for the second eye, documentation of symptoms affecting quality of life that cannot be improved 
with glasses is necessary, especially if visual acuity in the eye with cataract is better than 20/40 (Centers for Medicare \& Medicaid Services, 2016). The significant effect of cataract in an untreated eye shown in our study could serve as an indicator of the need for cataract surgery for the second eye. In the absence of the second eye surgery, patients should be made aware of the severe impact of the HLG found in this study. If patients with unilateral cataract have any reason to believe (such as comments from passengers, or their own observations of visual difficulty when they are in the passenger seat) that their driving performance is affected by headlight glare, they should request second eye cataract surgery and limit night driving as much as possible.

\section{ACKNOWLEDGMENTS}

Supported in part by NIH grant R01EY024075 and NIH core grant P30EY003790.

\section{REFERENCES}

Agramunt, S., Meuleners, L. B., Fraser, M. L., Chow, K. C., Ng, J. Q., \& Raja, V. (2018). First and second eye cataract surgery and driver self-regulation among older drivers with bilateral cataract: a prospective cohort study. BMC Geriatrics, $18(1), 51$. https://doi.org/10.1186/s12877-018-0743-1

Borzendowski, S. A. W., Sewall, A. A. S., Rosopa, P. J., \& Tyrrell, R. A. (2015). Drivers' judgments of the effect of headlight glare on their ability to see pedestrians at night. Journal of Safety Research, 53, 31-37. https://doi.org/10.1016/j.jsr.2015.03.001

de Waard, P. W., IJspeert, J. K., van den Berg, T. J., \& de Jong, P. T. (1992). Intraocular light scattering in age-related cataracts. Investigative Ophthalmology \& Visual Science, 33(3), $618-625$.

Gruber, N., Mosimann, U. P., Müri, R. M., \& Nef, T. (2013). Vision and night driving abilities of elderly drivers. Traffic Injury Prevention, 14, 477-485.

https://doi.org/10.7892/boris.38898

Hwang, A. D., \& Peli, E. (2013). Development of a headlight glare simulator for a driving simulator. Transportation Research Part C: Emerging Technologies, 32, 129-143. https://doi.org/10.1016/j.trc.2012.09.003

Hwang, A. D., Tuccar-Burak, M., Goldstein, R., \& Peli, E. (2018). Impact of Oncoming Headlight Glare With Cataracts: A Pilot Study. Frontiers in Psychology, 9. https://doi.org/10.3389/fpsyg.2018.00164

Centers for Medicare \& Medicaid Services (2016). Local Coverage Determination (LCD) for Cataract Extraction (L33954), The CMS Medicare Coverage Database Archive (https://localcoverage.cms.gov/mcd_archive/).

Owsley, C., Stalvey, B., Wells, J., \& Sloane, M. E. (1999). Older drivers and cataract: driving habits and crash risk. Journals of Gerontology Series A: Biomedical Sciences and Medical Sciences, 54(4), M203-M211.

Owsley, C., Stalvey, B. T., Wells, J., Sloane, M. E., \& McGwin, G. (2001). Visual risk factors for crash involvement in older drivers with cataract. Archives of Ophthalmology (Chicago, Ill.: 1960), 119(6), 881-887.

Wood, J. M., Tyrrell, R. A., Chaparro, A., Marszalek, R. P., Carberry, T. P., \& Chu, B. S. (2012). Even Moderate Visual Impairments Degrade Drivers' Ability to See Pedestrians at Night. Investigative Ophthalmology \& Visual Science, 53(6), 2586-2592. https://doi.org/10.1167/iovs.11-9083 